\title{
Equation of State for laboratory astrophysics applications
}

\author{
M. Cotelo • P. Velarde $\cdot$ A.G. de la Varga • \\ C. García-Fernández
}

\begin{abstract}
New improvements on the calculation of Equation of State (EOS) for laboratory astrophysics applications are presented. A new empirical multiplier for the EOS is included to the original quotidian equation of state (QEOS) model developed by More et al. (Phys. Fluids 31:3059, 1988) to adapt it to the available experimental data and $a b$ initio molecular dynamics simulation. This model is used to obtain EOS tables suited for an adaptive mesh refinement hydrodynamic code with radiation transport for high energy density plasmas simulations called ARWEN introduced by Ogando and Velarde (J. Quant. Spectrosc. Radiat. Transf. 71(2-6):541, 2001).
\end{abstract}

Keywords Equation of state - Shockwave experiments · Molecular dynamics

\section{Introduction}

The EOS is a mathematical relation between the state variables and the properties of the system. The most common state variables are the absolute temperature $(T)$ and the density $(\rho)$ and by means of the EOS the rest of the state functions of the system are known, like pressure $P=P(T, \rho)$ or energy $E=E(T, \rho)$.

In the simulation of many kinds of high-energy dynamic process like planetary and stellar interior evolution, LA experiments or shockwave propagation it is necessary to know the EOS of each material involved. Often these systems

\footnotetext{
M. Cotelo $(\triangle) \cdot$ P. Velarde $\cdot$ A.G. de la Varga .

C. García-Fernández

Instituto de Fusión Nuclear, Universidad Politécnica de Madrid,

Madrid, Spain

e-mail: cotelo@din.upm.es
}

reach very different states during their evolution, from very low temperatures to millions of kelvin and densities from of $\rho=10^{-3} \mathrm{~g} / \mathrm{cm}^{3}$ to several times the normal solid density and the EOS must cover all these states.

In Sect. 2 the basic EOS model is introduced and it continues with a deeper description of the new improvements in the EOS in Sect. 2.1. Also the data used to generate the EOS tables is discussed in Sects. 2.2 and 2.3. The process to obtain the improved EOS analytical data is described in Sect. 2.4. Finally conclusions are resumed in Sect. 3.

\section{Equation of State}

The behavior of matter is very different depending on the state of the system and the EOS must include all these diverse effects like phase transitions, dissociation, partial ionization or degeneration.

The starting point of the EOS described is the simple QEOS model introduced by More et al. (1988), where it is assumed that the electronic and ionic quantities are additive to sufficient accuracy. The QEOS model is based in the computation of the Helmholtz free energy $F$ and considers two additive components for this function plus a bonding correction. Applying the additive assumption the Helmholtz free energy is $F(T, \rho)=F_{e}\left(T_{e}, \rho\right)+F_{i}\left(T_{i}, \rho\right)+F_{b}\left(T_{i}, \rho\right)$ where $T_{e}$ is the electronic temperature and $T_{i}$ is the ionic temperature. The electronic part, $F_{e}$, uses the Thomas-Fermi model (Nikiforov et al. 2005) and depends on $T_{\epsilon}$, the calculation of the ionic component $F_{i}$ is based in a combination of simple but well-known rules (ideal gas model or the Lindemann melting law) all combined in a single analytic model developed by Cowan and described in Cranfill and More (1978). Finally, a bonding correction, $F_{b}$, is added to get the correct properties at normal conditions (room temperature 
and 1 bar of pressure). The QEOS model gives a complete set of thermodynamic properties of the system like pressure, energy or entropy and their derivatives respect to the temperature and density and also it can compute the charge state of the system $\bar{Q}(T, \rho)$.

A new emprirical correction is used in QEOS to introduce more adjustable parameters to the EOS model and then fit a larger range of experimental data. The use of this new empirical correction do not prevent the use of other correction as those described by Young and Corey (1995). This new empirical correction will increase the accuracy of the original QEOS and expands its domain of use keeping the advantages of the model: a general self-contained (no external data needed) EOS able to deal with mixtures that uses a consistent method.

The use of a global EOS model has the advantage that is easy to modify and introduce new fitting parameter to increase the flexibility of the method to generate EOS tables for any material with a fast and reliable implementation. Other EOS that mix several different models depending on the $T-\rho$ state, like SESAME (Johnson 1994), are very difficult to treat because they have to avoid any inconsistency in the transition between models (the EOS quantities must be smooth enough). Also it is difficult to establish a general methodology to treat all materials and the creation of adapted EOS tables for new material often takes a long time. Finally other different approaches to an analytical EOS can be found in Mihalas et al. (1988), Timmes and Arnett (1999) or Blinnikov et al. (1996).

\subsection{Empirical multiplier}

This EOS model is based on the computation of the Helmholtz free energy to assure the thermodynamic consistency as described by Menikoff and Plohr (1989). The rest of properties of the system are obtained applying the usual thermodynamic relations to the Helmholtz free energy $F$. The correction of the EOS is done by an empirical multiplier $f(T, \rho)$ over the Helmholtz free energy, $F$. If $\tilde{F}(T, \rho)$ is the modified Helmholtz free energy, then

$$
\begin{aligned}
& \tilde{F}(T, \rho)=f F \\
& \tilde{P}(T, \rho)=\rho^{2}\left(\frac{\partial \tilde{F}}{\partial \rho}\right)=\rho^{2}\left(f_{\rho} F+f F_{\rho}\right) \\
& \tilde{S}(T, \rho)=-\left(\frac{\partial \tilde{F}}{\partial T}\right)=\left(f+T f_{T}\right) S-f_{T} E \\
& \tilde{E}(T, \rho)=\tilde{F}+T \tilde{S}=\left(f-T f_{T}\right) E+T^{2} f_{T} S \\
& \tilde{C}_{v}(T, \rho)=f C_{v}+T f_{T T} E+\left(2 f_{T}+T f_{T T}\right) T S
\end{aligned}
$$

where the form used to denote derivatives is $X_{y}=\partial X / \partial y$. All properties and their derivatives depend on the temperature and density, including the multiplier.
The new multiplier include $T$ and $\rho$ dependence in order to fit several kinds of experimental data at the same time. The mathematical form of the multiplier $f(T, \rho)$ is

$f(T, \rho)=f_{\infty}-\alpha \exp \left[-\left(\frac{T}{T_{0}(\rho)}\right)^{\beta}\right]$

where the function $T_{0}$ takes the next form.

$T_{0}(\rho)=T_{00} \frac{\rho}{\rho_{\text {ref }}} \exp \left[-\frac{\rho}{\rho_{\text {ref }}}\right]$

and the meaning of $f_{\infty}$ corresponds to the limit of the multiplier for very high temperatures, $f(\infty, \rho)$.

The use of this empirical correction introduces five adjustable parameters in the EOS: $f_{\infty}, \alpha, \beta, T_{00}$ and $\rho_{\text {ref }}$. All these parameters have to be positive to obtain a stable and physically correct EOS. The QEOS model is accurate enough for high temperatures and no correction is needed in this regime. Then the first parameter is often $f_{\infty}=1$ to remove the correction for high $T$. Finally four parameters have to be chosen to define the empirical multiplier. The variation of the Helmholtz free energy produced by the multiplier is limited because $f(T, \rho)$ can only take values between $(1-\alpha)$ and 1 concluding that $F$ cannot be raised as can be seen in (1).

This mathematical formulation of the multiplier reproduces correctly the physical limits of the EOS according to the third law of thermodynamics or the ideal gas limit for very high temperature and low density. Also (6) and (7) do not introduce any artificial effect in the EOS: this function is smooth and always increases its value with $T$.

In order to choose a set of parameters for the empirical multiplier, the corrected EOS is compared to available experimental data and ab initio molecular dynamics simulations to estimate the error of the model and then select the set of parameters that minimizes this error. In Fig. 1 is shown the available experimental shockwave data for aluminum against a set of Hugoniot curves obtained from corrected EOS using different sets of multiplier parameters. The empirical multiplier increases the flexibility of the original QEOS to adjust the analytical data to experiments and molecular dynamics simulations.

The correction in the EOS can reproduce effects not treated by the QEOS theory like solid-solid phase transitions or dissociation of molecular gases. Also QEOS usually overestimates the pressure of the systems so a correction to reduce it is needed, specially for low temperatures. The Thomas-Fermi theory used to compute the electronic component of the Helmholtz free energy cannot consider nothing but atomic species (no binding theorem, Lieb 1981) and a correction for molecular gases is needed. 

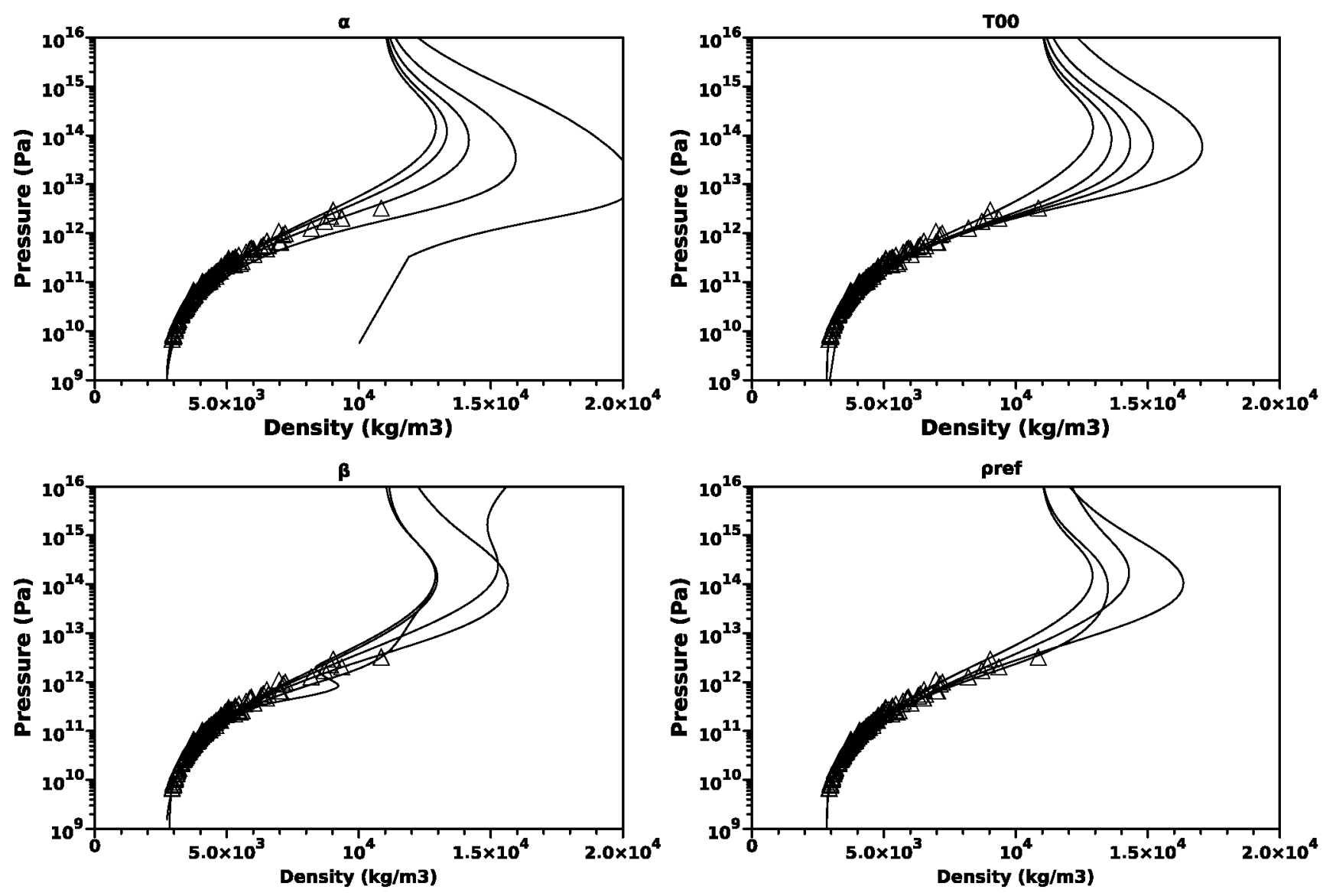

Fig. 1 Aluminum Hugoniot curves against experimental data

\subsection{Experimental data}

Almost every type of experimental data could be included in the fitting of the parameters but the most useful are the shockwave data, widely used for the study of mater under extreme conditions (Fortov et al. 2004; Huang 2002). This information covers a wide range of densities, from normal density to several times the solid density, and reach pressures of the order of GPa. There is available shockwave data in the scientific literature (Marsh 1980) for a great amount of materials of interest.

The EOS fitting against shockwave data is based in the Hugoniot relation:

$E-E_{0}=\frac{1}{2}\left(P+P_{0}\right)\left(\frac{1}{\rho_{0}}-\frac{1}{\rho}\right)$

Although the EOS model gives the thermodynamic properties as functions of temperature and density, it is possible to combine the pressure, $P=P(T, \rho)$, and energy, $E=E(T, \rho)$, to obtain the Hugoniot curve analytically, $P_{H}=P_{H}(\rho)$ and also the temperature along the Hugoniot, $T_{H}=T_{H}(\rho)$. To compute the Hugoniot curve is necessary

to know the initial state of the system, $\left(\rho_{0}, P_{0}, E_{0}\right)$, because it defines a concrete Hugoniot curve.

\subsection{Molecular dynamics simulations}

To improve the EOS estimations for states not reached by the shockwave experiments, it is possible to include simulations to the fitting process like ab initio molecular dynamics (AIMD) data and then adjust the multiplier parameters for warm dense matter and other states not covered by the experiments.

In $a b$ initio molecular dynamics the density functional theory (DFT) first developed by Hohenberg and Kohn (1964) and Kohn and Sham (1965) is used to calculate the forces on the nucleus solving the electronic structure of the system (see Martin 2004). Then the equations of motion of the atoms in the system are integrated, using the BornOppenheimer approximation or the Car-Parrinello method (Car and Parrinello 1985). This first principles technique are becoming a powerful tool for the computation of properties of matter like thermodynamic functions (Lambert et al. 2006), transport coefficients (Recoules et al. 2009; Laudernet et al. 2004) or phase transitions. But these methods are computationally very expensive and it is not possible 
to obtain EOS tables with enough density-temperature resolution directly from AIMD simulations. It is more useful to adjust a global EOS model with enough flexibility to the AIMD results and generate EOS tables from the simple analytical model.

In Fig. 2 the energy estimated by the corrected QEOS model is compared to AIMD data obtained from Recoules and Crocombette (2005). This AIMD simulation was done for a fixed density equal to the liquid density at the normal melting point and then this data allows to fit this EOS model for states close to phase transitions. In the same figure it is shown how the original QEOS model can fit the AIMD data with an error below $5 \%$ for high temperatures, but with a noticeable error (over $25 \%$ ) below $5000 \mathrm{~K}$. The correction of the EOS with the empirical multiplier proposed is able to reduce this error for low temperatures and to make the EOS reproduce this data.

Also in Fig. 3 is shown the experimental points of the Hugoniot curve $P_{H}(\rho)$ (Marsh 1980) for aluminum against the table 3717 of the SESAME database (Barnes and Lyon 1988 ) and the corrected EOS described. The densities and pressures represented in Fig. 3 correspond to liquid aluminum above $5 \mathrm{~g} / \mathrm{cm}^{3}$ and solid below this density. In squares are included the EOS obtained from AIMD simula- tions done by Recoules and Mazevet (2009) for six $\left(\rho_{i}, T_{i}\right)$ states that correspond to points of the Hugoniot computed with the SESAME table and in triangles the pressure of the corrected EOS for these same six states. The more accurate AIMD data estimates a pressure for the EOS higher than the analytical models, therefore QEOS and SESAME predict a

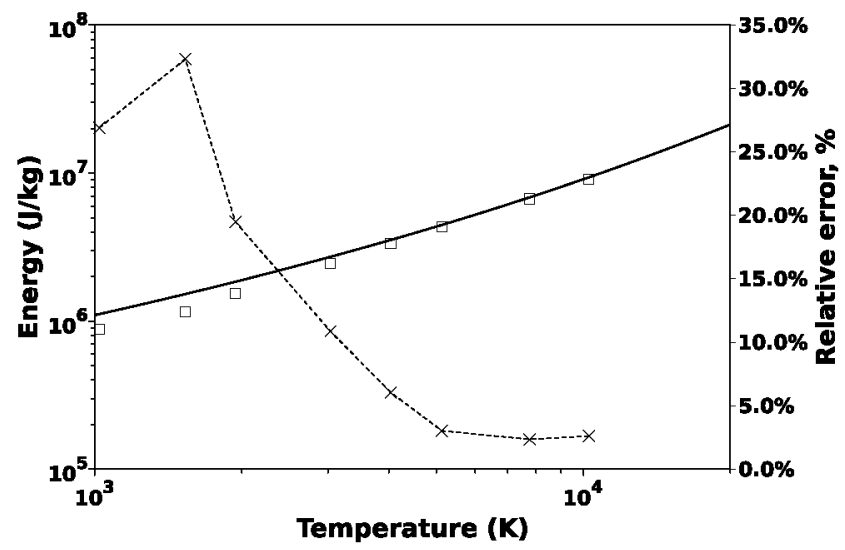

Fig. 2 Energy versus temperature for aluminum at fixed density of $\rho=2.35 \mathrm{~g} / \mathrm{cm}^{3}$ : full line is the original QEOS and in squares the AIMD data (Recoules and Crocombette 2005), left axis. Also the relative error between models is plotted (cross marks), right axis

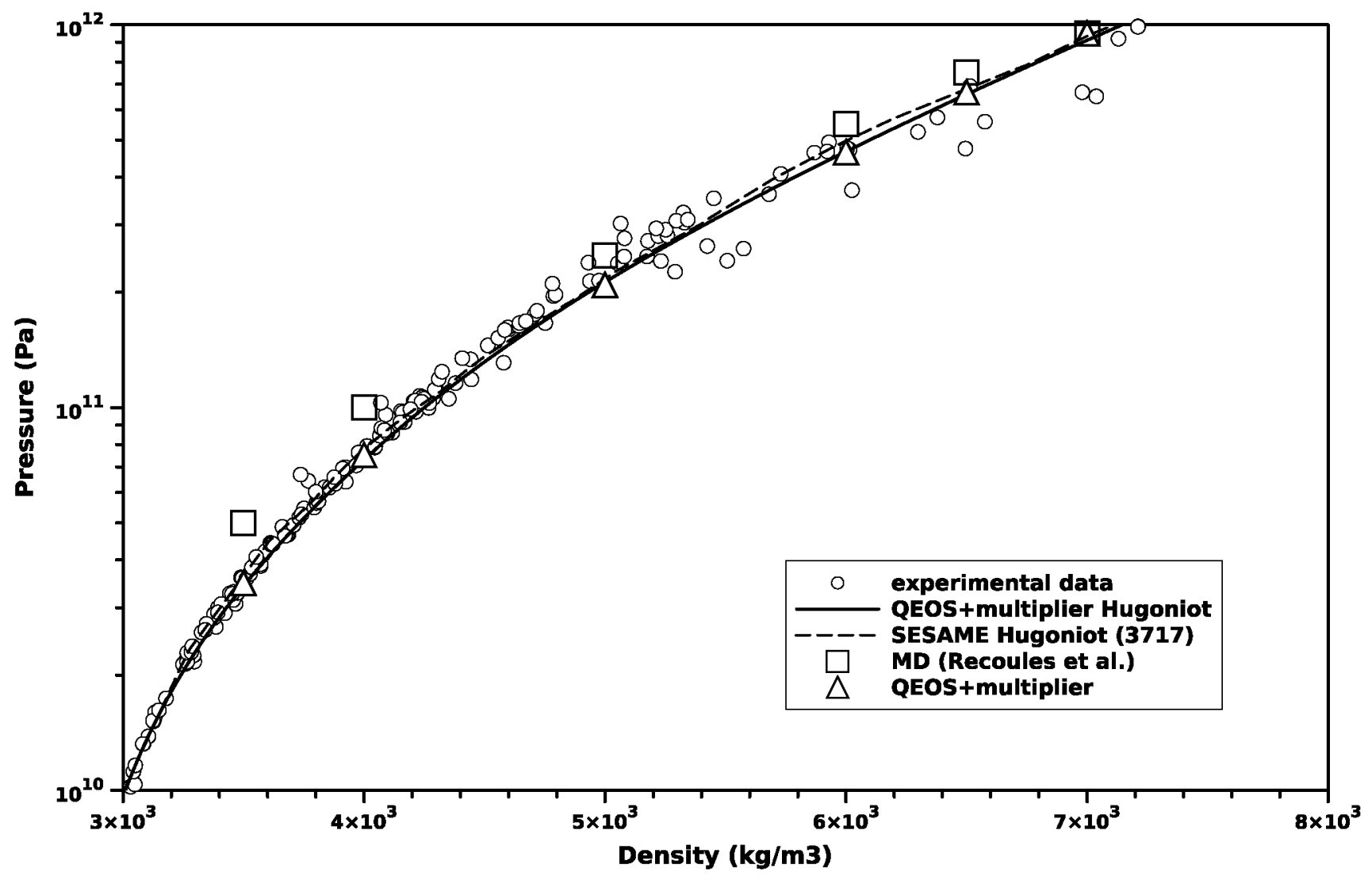

Fig. 3 Experimental Hugoniot data for aluminum (circles) against SESAME table 3717 (dashed) and the corrected EOS (full). In squares the EOS computed from points of the SESAME Hugoniot with AIMD (Recoules and Mazevet 2009) and in triangles the QEOS points 


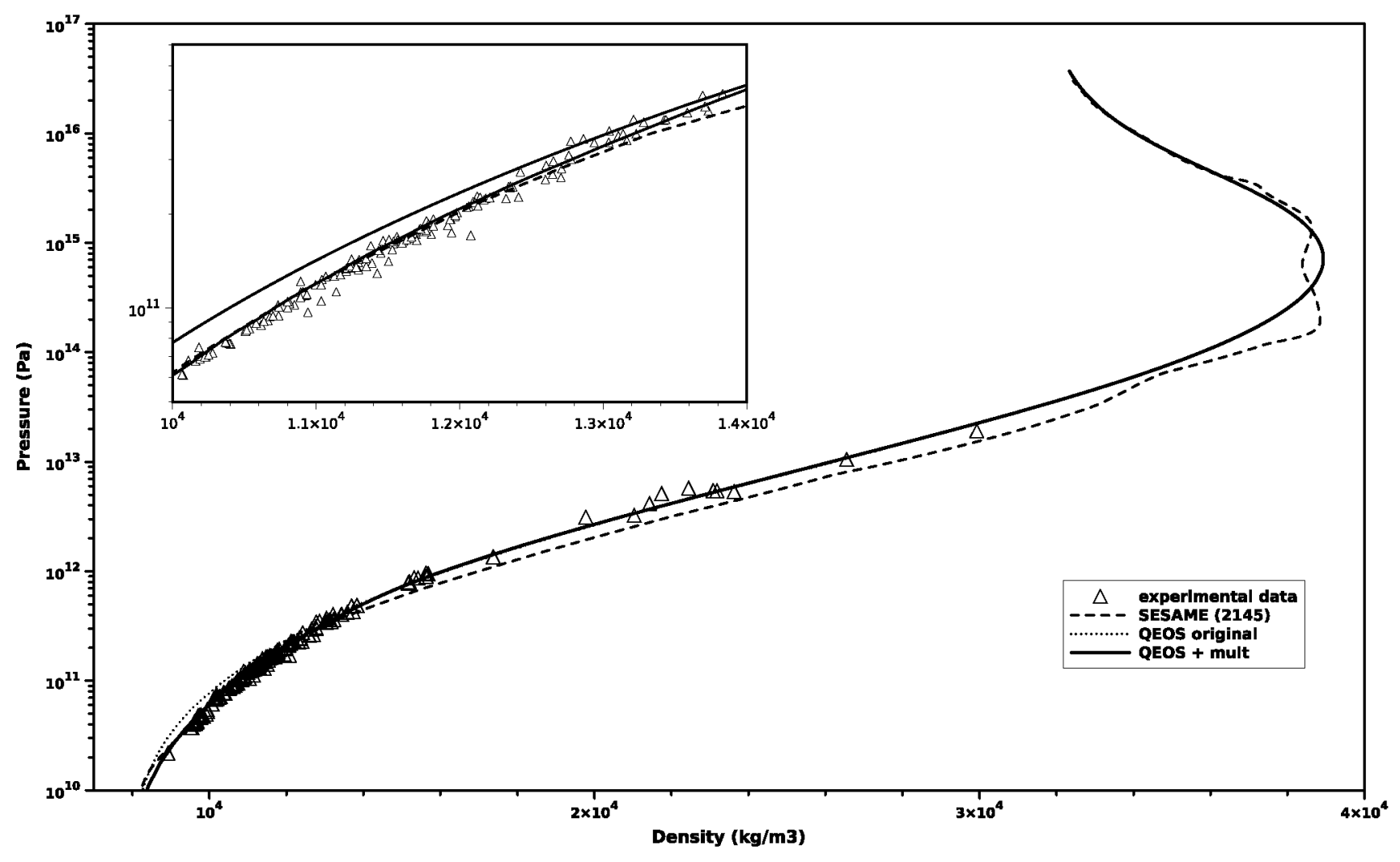

Fig. 4 Analytical Hugoniot curve using the corrected EOS proposed (full line) for Iron against the original QEOS model (doted-dashed line), SESAME database (dashed line) and experimental data (points)

higher temperature $T_{H}(\rho)$ for the Hugoniot in this regime. For higher pressures, the error between AIMD and the analytical models is reduced. The EOS computed from our own AIMD simulations also give lower temperatures than the corrected EOS for points of the Hugoniot curve like the results in Fig. 3.

\subsection{EOS fitting}

All this experimental and AIMD data are part of the input of a fitting procedure developed to search the multiplier parameters which minimize the mean quadratic error between the input data and the analytical EOS estimations of the same properties. In each error minimization step, the EOS consistency is checked to obtain correct values for the thermodynamic properties like positive $C_{v}$ and sound speed $c_{s}$ or correct limits for the entropy. This fitting procedure is quite flexible, reducing the error of the analytical Hugoniot to the experimental data as represented in Fig. 4. In this figure the iron Hugoniot was computed using three different references: the original QEOS model, the corrected EOS proposed in this work and the iron SESAME table 2145 created by Kerley et al. (1977). Also the available experimental (Marsh 1980) data for iron was compared with these models.

The use of the empirical multiplier reduces the quadratic mean error of QEOS against the experimental shockwave data in almost an order of magnitude compared with the original QEOS. Also it improves the estimations of SESAME tables (described by Johnson 1994) for the most of materials (Table 1). The corrected EOS with the empirical multiplier is able to reproduce the shock wave data with an improved accuracy for very different materials and all along the Hugoniot curve, as shown in Fig. 4.

The fitting procedure starts reproducing the shockwave and AIMD data with the original QEOS to establish an initial error. Then a first guess for the multiplier parameters is chosen and the multiplier is applied to the EOS tables to obtain a corrected EOS. This new EOS tables are compared to both experimental and AIMD data to compute the error of the analytical model.

$e^{2}=\frac{1}{N_{d}} \sum_{i=0}^{N_{d}}\left(\frac{X\left(T_{i}, \rho_{i}\right)-X_{\exp }\left(T_{i}, \rho_{i}\right)}{X_{\exp }\left(T_{i}, \rho_{i}\right)}\right)^{2}$

where $N_{d}$ is the number of experimental points and $X_{\exp }\left(T_{i}, \rho_{i}\right)$ is the $i$ th experimental data point for the state $\left(T_{i}, \rho_{i}\right)$. This formulation of the error is general and $X(T, \rho)$ could be the pressure along the Hugoniot curve for experimental data or the evolution of the energy depending on the temperature for a fixed density when AIMD data is used like in Fig. 2. Also the worth and reliability of the data can be 
Table 1 Root mean square error between analytical EOS models and shockwave experimental data

\begin{tabular}{lrlll}
\hline & Z & QEOS & This work & SESAME \\
\hline $\mathrm{Li}$ & 3 & $2.52 \times 10^{-1}$ & $1.93 \times 10^{-2}$ & $2.25 \times 10^{-2}$ \\
$\mathrm{Be}$ & 4 & $1.91 \times 10^{-2}$ & $2.20 \times 10^{-3}$ & - \\
$\mathrm{Al}$ & 13 & $2.49 \times 10^{-1}$ & $2.72 \times 10^{-2}$ & $7.42 \times 10^{-2}$ \\
$\mathrm{Fe}$ & 26 & $6.13 \times 10^{-2}$ & $2.60 \times 10^{-3}$ & $9.70 \times 10^{-3}$ \\
$\mathrm{Mo}$ & 42 & $1.60 \times 10^{-2}$ & $1.55 \times 10^{-2}$ & $4.37 \times 10^{-3}$ \\
$\mathrm{Au}$ & 79 & $1.88 \times 10^{-2}$ & $3.22 \times 10^{-3}$ & $7.05 \times 10^{-3}$ \\
$\mathrm{~Pb}$ & 82 & $8.15 \times 10^{-2}$ & $5.92 \times 10^{-3}$ & $2.38 \times 10^{-2}$ \\
\hline
\end{tabular}

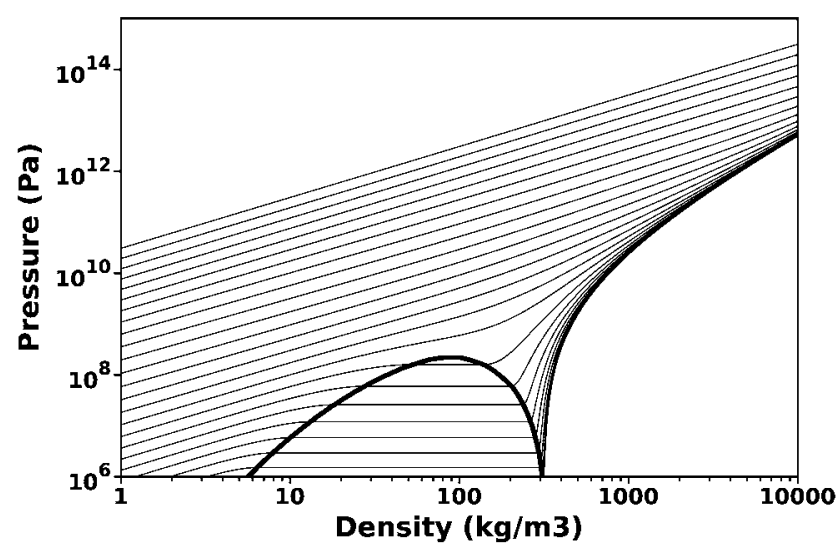

Fig. 5 Pressure versus density along isotherms for lithium. The lowest isotherm corresponds to $100 \mathrm{~K}$ and the highest to $6.45 \times 10^{6} \mathrm{~K}$

considered computing an error $e_{i}^{2}$ using (9) for each kind of data and give a statistical weights $\omega_{i}$ to each error and then average the mean error, $e^{2}=\sum \omega_{i} e_{i}^{2}$, where $\sum \omega_{i}=1$.

In the next step an iterative scheme starts to search the set of parameters that minimizes the error defined in (9). It begins computing four new EOS tables, each with the same base values for the parameters except one of them that has been modified. After that the AIMD and experimental data are reproduced with each of these four corrected EOS tables and the error between analytical estimations and input data are computed. Finally the procedure compares the error for each EOS table with the previous error and in consequence update the set of parameters. The iterative procedure ends when no improvements in the error can be achieved for variations of the multiplier lower than a defined tolerance.

When a set of multiplier parameters is chosen then suitable EOS tables for the selected materials are produced for LA applications. The EOS obtained with this method is processed to construct the liquid-vapor phase transition and includes this effect in the EOS tables to avoid instabilities in the thermodynamic properties. This phase transition is calculated using the usual mechanical and chemical equilibrium relations of the system. In Fig. 5 the lithium corrected EOS table is plotted. QEOS estimates a very high temper- ature for the critical point and for the example in Fig. 5 it corresponds to about $5000 \mathrm{~K}$ for lithium when the real value is $3223 \mathrm{~K}$.

\section{Conclusions}

Starting with simple models is possible to obtain an accurate EOS that reproduces the experimental and AIMD data for a wide range of densities and temperatures as seen in Fig. 4. The scheme described is general and can be applied for almost every kind of material, including mixtures. Also this procedure is easy to implement and computationally fast. It is important to treat the EOS in a thermodynamically consistent way to create suitable data for hydrodynamic codes.

The great developments in AIMD generate new possibilities to compute EOS properties with first-principle methods, obtaining accurate and reliable data. This allows to fit the global analytical EOS to different kinds of data. Using this scheme is possible to incorporate more fitting parameters to the formulation of the empirical multiplier $f(T, \rho)$ to increase the flexibility of the correction.

Acknowledgements The authors would like to thank the financial support provided by the Spanish Ministerio de Educación y Cultura program no. ENE2009-09837 and also to the European Commission projects HiPER-PP (211737) and ELI-PP (212105).

\section{References}

Barnes, J., Lyon, S.: SESAME EOS table 3717 for Aluminum (1988) Blinnikov, S.I., Dunina-Barkovskaya, N.V., Nadyozhin, D.K.: Astrophys. J. Suppl. Ser. 106 (1996)

Car, R., Parrinello, M.: Phys. Rev. Lett. 55(22), 2471 (1985)

Cranfill, C.W., More, R.M.: The Cowan theory is partially described in National Technical Information Service Document No. LA-7313MS. Technical report, Los Alamos National Laboratory (1978)

Fortov, V.E., Altshuler, L.V., Trunin, R.F., Funtikov, A.I.: High Pressure Shock Compression VII: Shock Waves and Extreme States of Matter. High Pressure Shock Compression. Springer, New York (2004). ISBN 0387205756

Hohenberg, P., Kohn, W.: Phys. Rev. 136(3B), B864 (1964)

Huang, Y.K.: A Short Treatise on Shock Waves and Equations of State. Nova Science (2002). ISBN 1590333241

Johnson, D.: The SESAME database. Technical report, Los Alamos National Laboratory, Group T-4 (1994)

Kerley, G., Barnes, J., Rood, J.: SESAME EOS table 2145 for Irion (1977)

Kohn, W., Sham, L.J.: Phys. Rev. 140(4A), Al 133 (1965)

Lambert, F., Clérouin, J., Zérah, G.: Phys. Rev. E 73(1), 016403 (2006)

Laudernet, Y., Clérouin, J., Mazevet, S.: Phys. Rev. B 70(16), 165108 (2004)

Lieb, E.H.: Rev. Mod. Phys. 53(4), 603 (1981)

Marsh, S.P.: LASL Shock Hugoniot Data. Scientific Laboratory Series on Dynamic Material Properties, vol. 5. University of California Press, Berkeley (1980). ISBN 0520040082

Martin, R.M.: Electronic Structure: Basic Theory and Practical Methods. Cambridge University Press, Berkeley (2004). ISBN 0521782856,9780521782852 
Menikoff, R., Plohr, B.J.: Rev. Mod. Phys. 61(1), 75 (1989)

Mihalas, D., Dappen, W., Hummer, D.G.: Astrophys. J. 331, 815 (1988)

More, R., Warren, K., Young, D., Zimmerman, G.: Phys. Fluids 31, 3059 (1988)

Nikiforov, A.F., Novikov, V.G., Uvarov, V.B.: Quantum-Statistical Models of Hot Dense Matter: Methods for Computation Opacity and Equation of State. Progress in Mathematical Physics, vol. 37. Birkhäuser, Basel (2005). ISBN 3764321830
Ogando, F., Velarde, P.: J. Quant. Spectrosc. Radiat. Transf. 71(2-6), $541(2001)$

Recoules, V., Mazevet, S.: Phys. Rev. B 80(6), 064110 (2009)

Recoules, V., Crocombette, J.P.: Phys. Rev. B 72(10), 104202 (2005)

Recoules, V., Lambert, F., Decoster, A., Canaud, B., Clérouin, J.: Phys. Rev. Lett. 102(7), 075002 (2009)

Timmes, F.X., Arnett, D.: Astrophys. J. Suppl. Ser. 125, 277 (1999)

Young, D.A., Corey, E.M.: J. Appl. Phys. 78(6), 3748 (1995) 